\title{
Searches for continuous gravitational waves with the LIGO and Virgo detector
}

\author{
Keith Riles ${ }^{1}$ \\ for the LIGO Scientific Collaboration and Virgo Collaboration \\ ${ }^{1}$ Physics Department, Univ. Michigan, 450 Church St., Ann Arbor, MI 48109-1040, U.S.A. \\ email: kriles@umich.edu
}

\begin{abstract}
The LIGO Scientific Collaboration and Virgo Collaboration have carried out joint searches in LIGO and Virgo data for periodic continuous gravitational waves. These analyses range from targeted searches for gravitational-wave signals from known pulsars, for which precise ephemerides from radio or X-ray observations are used in matched filters, to all-sky searches for unknown neutron stars, including stars in binary systems. Between these extremes lie directed searches for known stars of unknown spin frequency or for new unknown sources at specific locations, such as near the galactic center or in globular clusters. Recent and ongoing searches of each type will be summarized, along with prospects for future searches using data from the Advanced LIGO and Virgo detectors.
\end{abstract}

Keywords. Gravitational waves, Relativity, Instrumentation: interferometers, Stars: neutron, Pulsars

The hunt for gravitational waves has been a long one, carried out over decades, using instruments ranging from the Earth itself (normal mode excitation), to 1-ton metal bars to multi-km interferometers, to radio telescopes monitoring the precise timing of arrays of millisecond pulsars scattered across the sky. From the timing variations observed in the famous Hulse-Taylor binary pulsar system (Hulse \& Taylor 1975), one can be confident that gravitational waves are emitted with appreciable magnitude by astrophysical systems, but direct detection of those waves has proven to be an experimental challenge (see Riles 2012).

The focus of this article is the search in data from the LIGO (see Abbott et al. 2009) and Virgo (see Acernese et al. 2005) laser interferometers for evidence of continuous gravitational waves in the audio band, as might be radiated by nearby, rapidly spinning neutron stars. To emit gravitational waves, a spinning star must be characterized by some non-axisymmetry, e.g. , due to a "mountain" ( $\sim \mathrm{mm}$ high) at the star's equator or due to stellar precession. Because resulting strain amplitudes reaching the Earth are expected to be quite small ( $\sim 10^{-24}$ or much smaller), detection requires integration of data streams over long observation spans (the longest to date being $\sim 23$ months).

Because of computational cost considerations, it is natural to classify searches for continuous gravitational waves into three broad categories: 1) targeted - in which precise pulsar ephemerides from radio, X-ray or $\gamma$-ray observations can be used in a coherent integration over the full observation span; 2) directed - in which the direction of the source is known precisely, but for which little or no frequency information is known; and 3) all-sky - in which there is no information about location or frequency.

Targeted searches for known pulsars have been published based on data from the first five LIGO data runs (S1-S5) and on the second Virgo data run (VSR2), with continuing searches in the sixth LIGO run (S6) and the most recent Virgo run (VSR4). The S5 search determined upper limits (Abadie et al. 2010; based on 23 months of observation span) for 


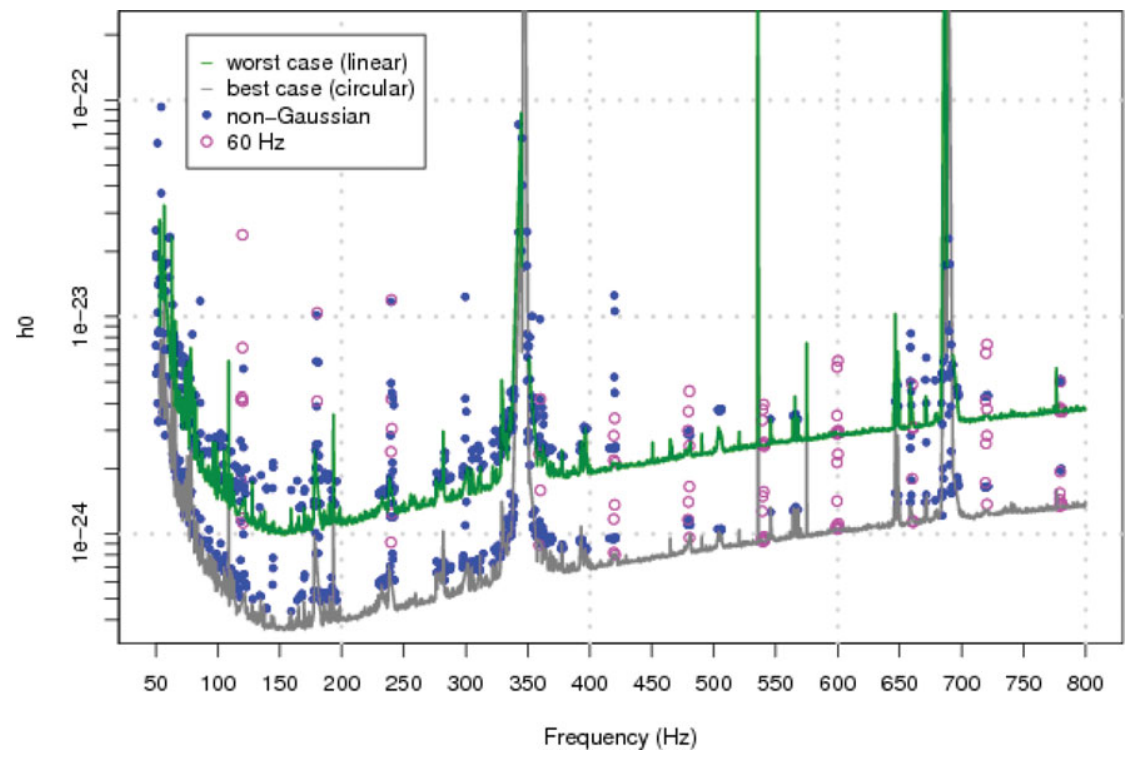

Figure 1. All-sky upper limits on the GW amplitude of unknown sources. The upper (green) curve shows worst-case upper limits (most unfavorable orientation of a linearly polarized source) in analyzed $0.25 \mathrm{~Hz}$ bands. The lower (gray) curve shows upper limits assuming a circularly polarized source. Solid points and circles denote bands with severe instrumental contaminations and hence unreliable limits.

116 known pulsars in the LIGO band. Highlights from the search included a lowest-strain upper limit of $2.3 \times 10^{-26}(\mathrm{~J} 1603-7202)$ and a lowest stellar ellipticity limit of $7 \times 10^{-8}$ (J2124-3358). In addition, a limit of $2 \%$ was placed on the fraction of rotational energy loss of the Crab pulsar that can be attributed to gravitational radiation. A more recent search (see Abadie et al. 2011) in Virgo VSR2 data for the Vela pulsar at an expected gravitational wave frequency of about $22 \mathrm{~Hz}$ (for which Virgo sensitivity is substantially better than LIGO's) yielded an upper limit of about $35 \%$ on Vela's fractional energy loss due to gravitational waves.

Because of computational costs of searching long observations times without a priori knowledge of gravitational wave frequency evolution, one must make tradeoffs in directed searches for particular objects or points in the sky. A published broadband search (see Abbott et al. 2010) for the X-ray-emitting compact central object in the Cassiopeia supernova remnant provides one example, based on analysis of a subset of LIGO S5 data (time span of $\sim 12$ days), for which the lowest strain upper limit was $7 \times 10^{-25}$ at $\sim 150$ $\mathrm{Hz}$. Because Cas A is only about 300 years old, this search incorporated a search over spin frequency and over its 1 st and 2 nd time derivatives. The resulting large parameter space volume for even a single point on the sky led to degraded strain sensitivity, compared to that achieved in the targeted searches described above.

All-sky searches for unknown neutron stars must cope with a still larger parameter space volume (as quantified by number of distinct templates searched for a fixed maximum SNR mismatch). Figure 1 shows all-sky strain upper limits (Abadie et al. 2012) on spinning isolated neutron stars, based on analysis of the full S5 data set, using semicoherent sums of Doppler-demodulated Fourier transform powers from tens of thousands of half-hour intervals ("PowerFlux" algorithm). A complementary and wider-band search of S5 data, based on Fourier transforms of longer coherence time (up to 25 hours per 
interferometer) and using the Einstein@Home distributed-computing project, led to comparable sensitivity in a search recently submitted for publication (see Aasi et al. 2012).

Comparison of the targeted and all-sky strain upper limits shown in figure 1 confirms the expected (substantial) degradation of sensitivity for searches that must search large parameter space volumes and hence must set high SNR thresholds, to cope with otherwise increased statistical outlier counts. For this reason, the "photon astronomer" community is encouraged not only to search for new and exotic objects that could serve as potential gravitational wave candidates, e.g. , nearby pulsars with high rotational energy losses, but also to determine spin rotations for known objects, such as Cas A and Scorpius X-1.

Installation of Advanced LIGO and Advanced Virgo has begun, with early operation expected circa 2015. When these detectors reach design sensitivity near the end of the decade, strain amplitude sensitivities and hence ranges within the galaxy will improve by an order of magnitude. Electromagnetic measurements could well make the difference between discovering and missing a star with a detectable gravitational wave signal.

\section{Acknowledgements}

The authors gratefully acknowledge the support of the United States National Science Foundation for the construction and operation of the LIGO Laboratory, the Science and Technology Facilities Council of the United Kingdom, the Max-Planck-Society, and the State of Niedersachsen/Germany for support of the construction and operation of the GEO600 detector, and the Italian Istituto Nazionale di Fisica Nucleare and the French Centre National de la Recherche Scientifique for the construction and operation of the Virgo detector. The authors also gratefully acknowledge the support of the research by these agencies and by the Australian Research Council, the International Science Linkages program of the Commonwealth of Australia, the Council of Scientific and Industrial Research of India, the Istituto Nazionale di Fisica Nucleare of Italy, the Spanish Ministerio de Economía y Competitividad, the Conselleria d'Economia Hisenda i Innovació of the Govern de les Illes Balears, the Foundation for Fundamental Research on Matter supported by the Netherlands Organisation for Scientific Research, the Polish Ministry of Science and Higher Education, the FOCUS Programme of Foundation for Polish Science, the Royal Society, the Scottish Funding Council, the Scottish Universities Physics Alliance, The National Aeronautics and Space Administration, the National Research Foundation of Korea, Industry Canada and the Province of Ontario through the Ministry of Economic Development and Innovation, the National Science and Engineering Research Council Canada, the Carnegie Trust, the Leverhulme Trust, the David and Lucile Packard Foundation, the Research Corporation, and the Alfred P. Sloan Foundation.

\section{References}

Aasi, J., et al. 2012, submitted to Phys. Rev. D, arXiv:1207.7176

Abadie, J., et al. 2010, ApJ 713, 671

Abadie, J., et al. 2011, ApJ 737, 93

Abadie, J., et al. 2012, Phys. Rev. D 85, 022001

Abbott, B., et al. 2009, Rep. Prog. Phys. 72, 076901

Abbott, B., et al. 2010, ApJ 722, 1504

Accadia, T., et al. 2012, J. Inst. 7, P03012

Hulse, R. A. \& Taylor, J. H. 1975, ApJ 195 L51

Riles, K. 2012, to appear in Prog. Part. Nuc. Phys., arXiv:1209.0667 